\title{
Darstellungsformen von Medizingeschichte im Fernsehen
}

\author{
Joan Kristin Bleicher
}

Im Zentrum dieses Beitrags steht eine Untersuchung der inbaltlichen und formalen Darstellungscharakteristika der Thematisierung von Medizingeschichte in unterschiedlichen fiktionalen und nonfiktionalen Sendeformen des Fernsebens. Der Text berücksichtigt dabei allgemeine Vermittlungskonventionen des Fernsebens, den Einfluss von Senderstrategien auf die vermittelten Inhalte und Wechselwirkungen zu Traditionslinien der Geschichtsvermittlung anderer populärer Erzäblweisen aus den Bereichen Literatur und Film. Literatur und Film stellen Rollenmuster des Arztes bereit, die der Personalisierung medizinhistorischer Themen im Fernsehen dienen. Der Überblick zum Genrespektrum und den Darstellungskonventionen von Medizingeschichte zeigt grundlegende Themen, Dramaturgien und Darstellungsmittel, die die Darstellung von Medizin in den Medien bestimmen.

Keywords: Medizingeschichte, Gesundheitskommunikation, Fernsehästhetik, fiktionale Genres, Dokumentation

Der Bereich aktueller Thematisierung von Medizin ist bislang vor allem für fiktionale Fernsehsendungen in den USA und Deutschland ${ }^{1}$ untersucht worden. Die Thematisierung historischer Ereignisse im Erzählsystem des Fernsehens besitzt generell das Potenzial der Kollektivierung von Erinnerung, die sich ständig neu aus einer immer weiter wachsenden Zahl medial vermittelter historischer Beiträge konstituiert. Der Beitrag gibt am Beispiel des Themenkomplexes „Medizingeschichte“ einen Überblick über unterschiedliche Sende- und Darstellungsformen von Geschichte im Fernsehen. Im Zentrum der Analyse stehen die jeweils vermittelten Rollenstereotypen und ihre spezifischen Wirkungspotenziale. Dabei zeigen Bezüge zur Darstellung von Medizingeschichte im Spielfilm grundlegende Themen und Dramaturgien, die medienübergreifend die Darstellung von Medizin beeinflussen und Schemata herausbilden, die die Wahrnehmung der Rezipienten bestimmen.

Der Beitrag verweist zunächst auf die Struktur des Fernsehens als Erzählsystem, das in unterschiedlichen Bereichen seines Programmangebots die Komplexität der Wirklichkeit in kausale Handlungsmuster überführt. Im folgenden Abschnitt werden unterschiedliche Einflüsse der Kulturgeschichte auf die mediale Darstellung von Medizin vorgestellt. Die Beschäftigung mit den Charakteristika der Präsentation historischer Inhalte im Fernsehen und ihren Funktionen zeigt die Art und Weise, wie die Thematisierung von Medizingeschichte von allgemeinen Senderstrategien beeinflusst wird, die Abhängigkeit der Thematisierungen von Medizingeschichte von den Darstellungskonventionen der Sendeformen und die Wechselwirkungen aus fiktionalen und dokumentarischen Darstellungselementen. Anschließend werden grundlegende formale und in-

1 So etwa in den Beiträgen des Augenblick Hefts 28, 1998, zu Krankenhausserien im Fernsehen und bei Rossmann, Constanze (2002): Die heile Welt des Fernsehens. Eine Studie zur Kultivierung durch Krankenhausserien, München. Vergleiche auch den Beitrag von Rossmann in diesem Themenheft. 
haltliche Vermittlungsformen in den unterschiedlichen Sendeformen beschrieben. Insgesamt werden auf unterschiedlichen Vermittlungsebenen formale und inhaltliche Aspekte der Darstellung von Medizingeschichte thematisiert.

\section{Fernsehen als Archiv des Gesellschaftswissens}

Historie und Erzählung sind traditionell eng verbunden. Historiker wie Reinhart Kosselek und Hayden White ${ }^{2}$ zeigen, dass Geschichte, auch Medizingeschichte, erst selbst zur Geschichte werden muss, bevor sie vermittelt und erinnert werden kann. Da die Weltvermittlung durch Erzählungen zu den Grundbedürfnissen der Menschen gehört, sind diese Erzählungen ein zentraler Faktor der Kulturgeschichte. Als komplexe Erzählsysteme sammelten bereits unterschiedliche Mythologien das Weltwissen und das kollektive Gedächtnis ihres Kulturraumes in einem Ensemble unterschiedlicher Erzählungen, die jeweils Teilbereiche dieses Wissens erläuterten und sich den jeweils aktuellen Anforderungen anpassten. ${ }^{3}$ Dieses Verfahren wurde von der Literatur und den Massenmedien übernommen: Gerhart von Graevenitz hat gezeigt, dass sich die Printmedien als Träger und Vermittler des kollektiven Gedächtnisses der Gesellschaft etablierten. ${ }^{4}$ Ende des 19. Jahrhunderts begann der Film, als Gedächtnisraum zu fungieren, Eike Wenzel untersuchte den Film als Ort der Gedächtnisfiguration. ${ }^{5}$ Im 20. Jahrhundert übernahm das Fernsehen diese Funktion. In den unterschiedlichen Bereichen seines Programmangebots überführt das Fernsehen die unüberschaubare Komplexität von Wirklichkeit in überschaubare Handlungsabfolgen, in denen die Ereignisse an die Intention von Personen gebunden sind. Das mediale Erzählsystem Fernsehen ${ }^{6}$ fungiert als zentraler Faktor der Kontingenzreduktion.

„Television is a centralized system of storytelling. Its drama, commercials, news, and other programs bring a relatively coherent system of images and messages into every home. (...) Television has become the primary common source of socialization and everyday information (mostly in the form of entertainment) of otherwise heterogeneous population."7

Durch seine kombinatorische Nutzung populärer Erzählweisen unterschiedlicher kulturhistorischer Herkunft erreichte das Fernsehen seine derzeitige Position als Leitmedium innerhalb des Mediensystems. Die aus diesen kombinatorischen Verfahren entstehende besondere Vielfalt von Sendungsangeboten ermöglicht es dem Fernsehen, als Er-

2 Siehe dazu beispielsweise: White, Hayden (1990): Die Bedeutung der Form. Erzählstrukturen in der Geschichtsschreibung, Frankfurt am Main.

3 Vergleiche hierzu: Assmann, Jan (1988): Kollektives Gedächtnis und kulturelle Identität. In: Jan Assmann/Tonio Hölscher (Hrsg.), Kultur und Gedächtnis, Frankfurt am Main, S. 9-19.

4 Graevenitz, Gerhard von (1993): Mythos. Zur Geschichte einer Denkgewohnheit. Stuttgart 1987 und derselbe: Memoria und Realismus. Erzählende Literatur in der deutschen Bildungspresse des 19. Jahrhunderts. In: Anselm Haverkamp/Renate Lachmann (Hrsg.), Memoria. Vergessen und Erinnerung, München, S. 283-304.

5 Wenzel, Eike (2000): Gedächtnisraum Film. Die Arbeit an der deutschen Geschichte in Filmen seit den sechziger Jahren, Stuttgart.

6 Vergleiche hierzu: Bleicher, Joan Kristin (1999): Fernsehen als Mythos. Poetik eines narrativen Erkenntnissystems. Opladen.

7 Gerbner, G.; Gross, L.; Morgan, M. \& Signorielli, N. (1986): Living with television: The dynamics of the cultivation process. In: J. Bryant/D. Zillmann (eds.), Perspectives on media effects. Hillsdale, NJ: Erlbaum, S. 18. 
zählsystem vielfältige Funktionen für die Gesellschaft, aber auch für den einzelnen Zuschauer, zu übernehmen.

Die Thematisierung historischer Ereignisse im System der Fernseherzählungen besitzt das Potenzial der Kollektivierung von Erinnerung, die sich ständig neu aus einer immer weiter wachsenden Zahl medial vermittelter historischer Beiträge konstituiert. Das Fernsehen sichert sich sowohl in seiner ständigen Aktualisierung als auch in der ständigen Thematisierung des Vergangenen seine zentrale Position als kollektive Gedächtnismaschine. Das Fernsehen ist zentraler Faktor in der allgemeinen Funktion des Mediensystems, als Archiv für die jeweils aktuell vermittelte Wirklichkeit zu fungieren. ${ }^{8}$ Es lenkt in seiner dauerhaften und vielfältigen Vermittlung von Geschichte in unterschiedlichen Sendeformen die Blickrichtung auf jene Ereignisse, die aus der Perspektive der Gegenwart als historische Wissensbestände einer Gesellschaft für die Zukunft bedeutsam erscheinen. Eine fehlende thematische Integration von historischen Ereignissen in die Berichterstattung des Leitmediums Fernsehen führt auch zum drohenden Verlust dieses Erinnerungsbestandteils des kollektiven Gedächtnisses.

Das Fernsehen stellt in den Erzählungen seiner Informationssendungen täglich aktualisierte Erinnerungselemente für das kollektive Gedächtnis der Gesellschaft bereit. ${ }^{9}$ Diese Erinnerungselemente lassen sich für die Einordnung und Bewertung aktueller Ereignisse nutzbar machen. Das Bedürfnis nach medial vermittelten Orientierungsmodellen der Vergangenheit steigt bei den Zuschauern mit den steten Veränderungen der Gegenwart, die ihre Alltagsgestaltung beeinflussen. Anhand der Vermittlung von Medizingeschichte lassen sich die Strukturprinzipien der allgemeinen Konstruktion von Geschichte durch das Fernsehen verdeutlichen.

Als eine Addition aus unterschiedlichen visuellen Erfahrungsräumen ist das Fernsehprogrammangebot für den Zuschauer jederzeit zugänglich. Die serielle Vermittlungsweise des Programmangebots, das mit der regelmäßigen Wiederkehr vergleichbarer Sendeformen den Zuschauern ständig zur Rezeption zur Verfügung steht, garantiert die dauerhafte Konstruktion der Linearität historischer Entwicklungen. Der kontinuierlich erfolgende Rückgriff auf Ereignisse der Vergangenheit bildet eine Folie, auf der gegenwärtige Veränderungen darstellbar und somit auch in der Bewertung ihrer Bedeutung leichter zu verstehen sind. Nachrichtensendungen und Magazine liefern durch ihre Rückblicke auf vergangene Entwicklungen Erklärungsmuster für aktuelle Ereignisse, die wiederum in der Anschlusskommunikation Teil des Rückblicks des nächsten aktuellen Ereignisses sind. Durch diesen kontinuierlichen Rückgriff auf vergangene Ereignisse findet bereits in der aktuellen Berichterstattung eine kontinuierliche Geschichtsschreibung durch das Medium Fernsehen statt.

\section{Medizingeschichte im Rahmen unterschiedlicher Senderstrategien}

\subsection{Medizingeschichte in kommerziellen Programmen}

Die Präsentation des Themenkomplexes Medizingeschichte folgt nicht nur den allgemeinen Konstruktionsprinzipien des Fernsehens, sondern ist auch in die Platzierungs-

8 Vergleiche hierzu: Bleicher 1999 (Fn. 6), S. $89 f$.

9 Vergleiche hierzu auch: Bleicher, Joan Kristin; Hickethier, Knut (1998): Die Inszenierung der Information im Fernsehen. In: Herbert Willems/Martin Jurga (Hrsg.), Inszenierungsgesellschaft. Ein einführendes Handbuch. Opladen, S. 369 - 384. 
und Gestaltungsstrategien verschiedener Senderstrategien eingebunden. Ins Angebot kommerzieller Vollprogramme erhalten Themen in der Senderkonkurrenz der neunziger Jahre nur dann Einzug, wenn sie den generellen Aufmerksamkeitsstrategien der populären Aufbereitung von Inhalten entsprechen: Hierzu gehört die Präsentation sensationeller Aufnahmen, ein attraktiver, heldenhafter Protagonist und/oder ein wirkungsvoll leidender Kranker, mit dem sich der Zuschauer identifizieren kann bzw. mit dem der Erlebniswert einer emotionalen Betroffenheit ausgelöst wird. Der Bezug abstrakter medizinhistorischer Inhalte auf konkrete Krankheitsbilder ermöglicht die Ansprache eines Zielpublikums aus Betroffenen.

Aus der strategischen Sicht der Programmplanung sichert die Thematisierung weit verbreiteter Krankheiten hohe Marktanteile. Der Themenkomplex Medizingeschichte ist daher auch in die Vermittlungsformen des Informationsbereichs der kommerziellen Anbieter integriert. Innerhalb von Magazinsendungen ist bei den kommerziellen Anbietern die faktische Darstellung des Historischen in eine emotionalisierende Dramaturgie eingebunden und wird so unmittelbar in das gegenwärtige Erleben der Zuschauer überführt. Faktenwissen aus Statements von Experten wird durch tränenreiche Erzählungen persönlicher Schicksale der Betroffenen selbst ergänzt. Auch das Spektakuläre bei der Thematisierung seltener Krankheiten ebenso wie als Element der visuellen Darstellung kommt bei der Präsentation des Historischen wie der gegenwärtigen Medizin nicht zu kurz. Die Programmverantwortlichen greifen gerne auf besonders spektakuläre Krankheitsbilder, wie etwa die grotesk elefantösen Gliedmaßen von Lymphdrüsenerkrankten, zurück. ${ }^{10}$ So erlebt die vergessen geglaubte Zurschaustellung Kranker auf den Jahrmärkten ihre Renaissance in diesen allabendlichen „Freakshows“ der Boulevardmagazine.

Populärwissenschaftliche Magazine wie „Galileo“ (Pro Sieben) nutzen die subjektive Betroffenheit durch das Thema Krankheit als Aufmerksamkeitsfaktor für den Themenkomplex medizinischer Forschung. Für den Zuschauer werden komplexe Zusammenhänge in einfache, verständliche Berichte überführt, die Medizingeschichte nutzen, um in gleich bleibenden Geschichten über unermüdliche Forscher vom permanenten Fortschritt der Wissenschaft zu erzählen.

\subsection{Medizingeschichte in öffentlich-rechtlichen Kulturprogrammen}

Anders ist das Erscheinungsbild medizinhistorischer Angebote in den Kulturprogrammen Arte oder 3sat, die in ihren Senderstrategien den Zielsetzungen von Informationsvermittlung und Bildung folgen. Hier wechseln Elemente der Zuschaueridentifikation mit unterschiedlichen Rollenmustern von Arzt oder Patient und dokumentarischen Thematisierungen von Forschungsentwicklungen. In dem monothematischen Strukturprinzip des Themenabends, etwa von Arte über Louis Pasteur, ergänzen sich die unterschiedlichen fiktionalen und dokumentarischen Vermittlungsformen zu einem Gesamtbild, das sowohl Fakten, den Menschen als auch seine Arbeit einschließt. Die Person des Arztes Pasteur wird in den Kontext des Medizinsystems seiner Zeit eingebunden. An die Seite des Erlebniswertes tritt so, dem Kulturanspruch des Senders entsprechend, ein Informationswert. Das konzentrische Programmmodell des Themenabends ermöglicht

10 Solche Kranken wurden wiederholt in Beiträgen der Boulevardmagazine von RTL und Sat.1 präsentiert. 
eine differenzierte Auseinandersetzung mit unterschiedlichen Aspekten des Gegenstandsbereichs und greift auf ein breites Spektrum dokumentarischer und fiktionaler Vermittlungsformen zurück.

\section{Darstellungskonventionen und inhaltliche Stereotypen}

Trotz der unterschiedlichen Zielsetzungen der Sendeanstalten lassen sich durchaus vergleichbare Darstellungskonventionen und inhaltliche Stereotypen der Vermittlung von Medizingeschichte im Fernsehen beobachten. Der Themenkomplex ist in die allgemeine Angebots- und Wirkungsstruktur des Mediums eingebunden. Die Nutzung bestehender Darstellungs- und Handlungsmuster unterschiedlicher kulturhistorischer Traditionslinien ist ein grundlegendes Verfahren der Fernsehvermittlung, das auch die Vermittlung von Geschichte beeinflusst. Von entscheidender Bedeutung ist dabei die Integration von Geschichtsbildern des Spielfilms. Produktionen aus unterschiedlichen Phasen der Filmgeschichte und damit Thematisierungen von unterschiedlichen Teilbereichen der Geschichte werden in den Fernsehprogrammen in zahllosen Wiederholungen reproduziert. Durch diese ständigen Wiederholungen wird der Zuschauer auch kontinuierlich mit formalen und inhaltlichen filmischen Stereotypen konfrontiert. Fernsehprogramme fungieren durch die Wiederholung von Spielfilmen als Bildwiederaufbereitungsanlagen ebenso wie als Erzählmaschinen, das Fernsehen bedient sich bei der Vermittlung von Geschichte der bereits vorhandenen Erzählungen und Bildmotive der filmischen Vermittlung von Geschichte. Der Historiker Arnold Sywottek hat in einem Vortrag zum Verhältnis von „Film und Geschichte“ darauf hingewiesen, wie sehr diese Erzählelemente und Bildmotive unsere Vorstellungen von Geschichte beeinflussen. ${ }^{11}$

Eine besondere Möglichkeit der Vermittlung von Geschichte im Fernsehen liegt durch die Vielfalt der genutzten Vermittlungsformen in ihrem vielschichtigen Informationsgehalt, Erlebniswert und in ihrer sinnlichen Erfahrbarkeit. Die televisionäre Konstruktion von Medizingeschichte nutzt Struktur- und Darstellungselemente der Erzählung. Dazu zählen eine kausale Struktur der Ereignisse, die durch die Bindung der Handlung an Zielsetzungen und Taten einzelner Personen erfolgt. Entwicklungen im Bereich der medizinischen Forschung werden durch die Verwendung erzählerischer Darstellungsmittel als Ergebnis des intentionalen Handelns einzelner Forscher beschrieben, die in einer Kette aufeinander aufbauender Untersuchungen entwickelt wurden. Ökonomische Einflüsse und Fehlentwicklungen der Forschung sind in dieser Form der narrativen Geschichtsvermittlung blinde Flecken, die nicht thematisiert werden.

Die narrative Konstruktion der Geschichte folgt in den unterschiedlichen Vermittlungszusammenhängen dem grundlegenden Handlungsmuster populärer Erzählungen mit der Abfolge: Harmonie, Störung der Harmonie, Konflikt, Konfliktlösung, Wiederherstellung der Harmonie. Im Vergleich zum Angebotsspektrum literarischer Erzählungen, etwa zum Genre des Historischen Romans, lässt sich Geschichte in Film- und Fernseherzählungen in Bild und Ton als direkter, unmittelbarer Eindruck vermitteln. ${ }^{12}$

11 Sywottek, Arnold (1997): Film und Geschichte. In: Knut Hickethier/Eggo Müller/Rainer Rother (Hrsg.), Der Film in der Geschichte. Dokumentation der GFF-Tagung, Berlin, S. 11 - 18.

12 Die Programmmacher planen die mögliche Wirkung der sinnlichen Erfahrbarkeit in Konzeption und Zielsetzung ihrer Sendungen ein. „Die ,sinnliche` Erfahrung von Geschichte soll gleichsam die Bereitschaft fördern, auch deren nüchterne Bilanz zu akzeptieren“, so Guido Knopp, Leiter der Redaktion Zeitgeschichte beim ZDF. 
Die Personenbindung der Fernsehvermittlung führt dazu, dass historische Prozesse als Ergebnis persönlicher Intentionen oder Wunschkonstellationen und nicht als Ergebnis faktischer Zusammenhänge und komplexer Gesellschaftsstrukturen geschildert werden.

\subsection{Einfluss kulturhistorischer Traditionslinien der Medizindarstellung}

In der über längere Zeiträume reichenden Vermittlung grundlegender Themen und gleich bleibender Dramaturgien medialer Erzählsysteme bildeten sich Schemata heraus, die die Wahrnehmung der Rezipienten bestimmen. Die Wiederholung gleichartiger Inhalte und gleich bleibender Darstellungsmittel auf der Angebotsseite konstruiert die Weltwahrnehmung der Rezeption. David Bordwell betont als Filmwissenschaftler die Bedeutung narrativer Schemata für die Strukturierung unserer Wahrnehmung. ${ }^{13}$

Auch das gegenwärtige Bild der Medizin in der Gesellschaft basiert auf Schemata, die in einer langen Entwicklungslinie kultureller und medialer Darstellung medizinischer Themen entstanden sind. Vertreter der Erzählforschung, wie Joseph Campbell, wiesen auf kulturübergreifend gleich bleibende Motive, Erzählabläufe, Rollenmuster und Darstellungsformen der narrativen Weltvermittlung hin, ${ }^{14}$ die auch die Vermittlung des Themenkomplexes Medizingeschichte bestimmen. Neben unterschiedlichen literarischen Vermittlungsformen haben vor allem die Darstellungsschemata des Spielfilms einen entscheidenden Einfluss auf die Präsentation von Medizingeschichte im Fernsehen. Hier sind die grundlegenden Rollenmuster der personengebundenen Darstellung festgelegt. Als Akteure treten Ärzte und Forscher auf, der Patient hingegen bleibt das im Vergleich passive Objekt der Handlung.

Gleich bleibende formale und inhaltliche Darstellungselemente haben im Verlauf der Kulturgeschichte Handlungs- und Rollen-Schemata entstehen lassen, die als Wahrnehmungsschemata die Raster der gegenwärtigen Erfahrung bilden. Bei den Schematabildungen innerhalb literarischer und medialer Vermittlungsformen sind Wechselwirkungen von Form und Inhalt auffällig, die im Folgenden am Beispiel der Thematisierung von Medizingeschichte analysiert werden.

\subsection{Rollenmuster des Arztes in kulturbistorischer Tradition}

Die kulturhistorische Traditionslinie der Thematisierung von Medizin weist eine Tendenz zur Personenbindung auf. Constanze Rossmann betont: „Das Bild des Arztes ist schon seit frühen Zeiten von besonderen Idealvorstellungen geprägt. Seit jeher war der Heilkundige eine zentrale Persönlichkeit in der Gesellschaft."15 In unterschiedlichen Bereichen der Kulturgeschichte, etwa der Literatur, kulminieren ganz unterschiedliche Themenkomplexe aus dem Bereich medizinischer Forschung und medizinischer Arbeit am Patienten um die Zentralfigur des Arztes. Die Figur des Arztes entspricht den Anforderungen mythologischer Erzählstrukturen, die James Chesebro wie folgt beschreibt: „In the mythical communication system, the central character is superior in kind to others both in terms of intelligence and in terms of his or her ability to control

13 Vergleiche hierzu: Bordwell, David (1985): Narration in the Fiction Film. London.

14 Campbell, Joseph (1978): Der Heros in tausend Gestalten. Frankfurt am Main.

15 Rossmann, 2002 (Fn. 1), S. 9. 
circumstances. “16 Populäre Erzählungen lassen den Arzt als potenziell allmächtigen Helfer und Heiler zum gottähnlichen Helden aufsteigen. Auf der Handlungsebene bleibt der Arzt durch die ständig wiederholte idealtypische Einfindung in das Schicksal des Patienten immer in der Nähe zum Menschen. Allmacht und Einfühlsamkeit bilden eine wirkungsvolle dramaturgische Mischung.

Der Arztbesuch dient nicht allein der Heilung, er nimmt auch Formen einer allgemeinen Lebensberatung an. Neben der Krankheit wird auch gleich die Lebenskrise mitbehandelt und notfalls auch der Antagonist des Patienten mittherapiert.

Neben das Rollenmuster des Heilers tritt das Rollenmuster des unermüdlich nach Wissen strebenden Forschers. Mit dem Ziel, die Wirkung von Spielfilmen zu optimieren, werden tradierte Figuren der Literaturgeschichte, wie der nach umfassenden Wissen strebende Faust, auf die „echten“ Forscher als Protagonisten übertragen.

Serielle Erzählweisen erleichtern die Stereotypenbildung. Bereits in der Trivialliteratur wurden Stereotypen der visuellen Präsentation der Ärzte „als große, schlanke, souveräne Schicksalsträger, denen von allen Seiten ein ehrfürchtiges Wohlwollen, ja eine geradezu religiöse Reverenz entgegengebracht wird“, ${ }^{17}$ entwickelt. Viele Arztserien des Fernsehens wie „Dr. Frank, der Arzt, dem die Frauen vertrauen“ oder „OP ruft Dr. Bruckner" sind direkt aus den seriell produzierten Heftchenreihen der Trivialliteratur hervorgegangen, so dass die Protagonisten vielen Fernsehzuschauerinnen bereits aus ihrer Lektüre vertraut waren. Das Fernsehen konnte so auf die bestehende Personenbindung von Rollenmustern der Trivialliteratur und populärer Filme aufbauen und nutzte sie für die Zuschauerbindung an seine Vermittlungsformen des Fiktions- und Informationsbereichs.

\subsection{Rollenmuster des Arztes als Spiegel gesellschaftlicher Wertekonstellationen}

In den die Fernsehvermittlung beeinflussenden literarisch und filmisch vermittelten Rollenmustern des Arztes spiegeln sich auch gesellschaftliche Wertekonstellationen wider. Klaus Kreimeier sieht im Rollenmuster des Arztes in den Spielfilmen der fünfziger Jahre eine Verkörperung gesellschaftlicher Stabilisierungsbedürfnisse, die eng an etablierte Rollenmuster der Präsentation von Autorität geknüpft sind. Aus seiner Sicht verbinden die Arzt-Filme „die philanthropisch-humanitären Momente der Gattung mit der angeblich ,volkstümlichen' Vorstellung, dass der Arzt Vater und schlafwandlerisch sicherer Feldherr, Magier und wissenschaftliche Kapazität in einem sei: Heilung durch die Autorität schlechthin widerfährt in diesen Filmen nicht nur dem einzelnen Patienten, sondern einer ganzen, unergründlicherweise an ihren eigenen Umständen erkrankten Zeit.“"18 In den fünfziger Jahren fungierten die Protagonisten von Filmen wie „Sauerbruch - das war mein Leben“ (1954) oder „Der Arzt von Stalingrad“ (1958) als potenzielle gesellschaftliche Vor- und Leitbilder. „Der Sauerbruch von 1954 ist das Muster-

16 Chesebro, James W. (1987): Communication, Values, and Popular Television Series - A Four Year Assessment. In: Horace Newcomb (Hrsg.), Television - The Critical View. New York, Oxford, S. 21f.

17 Zitiert nach: Rossmann, 2002 (Fn. 1), S. 15. Zu den Rollenmustern des Arztes in der Sekundärliteratur vergleiche auch: Hermand, Jost (1989): Kultur im Wiederaufbau. Die Bundesrepublik Deutschland 1945-1965. München, S. 373f.

18 Kreimeier, Klaus (1973): Kino und Filmindustrie in der BRD. Ideologieproduktion und Klassenwirklichkeit nach 1945. Kronberg, S. 97. 
bild der Autorität, wie der Untertan es sich wünscht: In schlafwandlerischer Sicherheit und auf Grund eines rätselhaften Geheimwissens wendet er jedes Leid zum Guten, sofern der Patient sich ihm nur vorbehaltlos anvertraut." ${ }^{19}$ Medizingeschichte transportiert durch diese Form der Personalisierung jeweils positiv besetzte Wertkonstellationen.

Auch das grundlegende Handlungsmotiv des Arztes als Heiler und Helfer der Patienten wird häufig auf aktuelle Gefährdungen der Gesellschaft ausgeweitet und so in seiner Bedeutungsdimension aufgewertet. Heimatfilme der fünfziger Jahre verbinden das medizinische Heilen mit ihren Wunschbildern einer heilen Welt, die zwar vom technischen Fortschritt und skrupellosen Geschäftemachern bedroht ist, deren Idylle im Kino aber immer wieder hergestellt werden kann. Auch die Genderkonstruktion dieser Filme spiegelt die Gefährdung zeitgebundener konservativer Lebensideale, sich ankündigende gesellschaftliche Veränderungen der Geschlechterrollen wurden im Verlauf der Filmhandlung wieder in bestehende Rollenmuster überführt: Die Landärztin des gleichnamigen Films gerät in Konflikt mit der Dorfgemeinschaft, die sich von keiner Frau behandeln lassen will und stattdessen lieber den männlichen Tierarzt konsultiert. In einer krisenhaft zugespitzten Situation darf sie schließlich ihr Können beweisen, kehrt jedoch mit der Heirat des Tierarztes glücklich in die tradierten Rollenmuster zurück. Die Bedrohung der Veränderung ist mit diesem Ende der Erzählung abgewendet.

Eine vergleichende Analyse ${ }^{20}$ einer Auswahl unterschiedlicher Sendungen zum Thema Medizingeschichte im Fernsehprogramm des Jahres 2001 zeigte, dass es in unterschiedlichen Genres verschiedene Bewertungen von Rollenmustern der Ärzte gibt, die auch vom Zeitbezug des jeweiligen Themas beeinflusst sind. Historische Arztgestalten werden in fiktionalen und dokumentarischen Rückblicken zu Halbgöttern stilisiert, während die Darstellung noch lebender Ärzte, etwa in Dokumentationen oder Magazinsendungen wie „Report“ oder „Panorama“, häufig das skrupellose Verhalten der Ärzte in den Mittelpunkt stellt. F. Nager beschreibt das Rollenmuster eines „zeitgemäßen“ Arzttyps „als einen methodengewandten, naturwissenschaftlichen Experten, der psychische und soziale Aspekte immer mehr außer Acht lässt. Das Mehr an Wissen geht mit einer Reduktion des Menschenbildes auf mechanistische Zusammenhänge einher und mündet in einer Entwertung des Patienten und auch der Persönlichkeit des Arztes. “21 Die selbstlose Forschungstätigkeit des traditionellen Rollenmusters vom Arzt als Forscher bildet den Gegenpol zum aktuellen Rollenmuster des skrupellosen Handlangers der Pharmaindustrie, der sinnlose Experimente an Patienten durchführt.

\subsection{Traditionslinien der visuellen Darstellung von Geschichte}

Zusätzlich zur Orientierung an etablierten Themen und Rollenmustern der Trivialliteratur kombiniert das Fernsehen auch Traditionslinien der visuellen Vermittlung von Geschichte. Mit seinem Darstellungsspektrum der Visualisierung historischer Fakten lehnt sich das Fernsehen an unterschiedliche kulturhistorische Traditionen der bildli-

19 Ulrich Gregor, Enno Patalas: Geschichte des Films. Reinbek 1976. Bd.2. S.420.

20 Die Verfasserin analysierte aus den Programmangeboten öffentlich-rechtlicher (ARD, ZDF, 3sat und arte) und kommerzieller Anbieter (RTL, Sat.1) formale und inhaltliche Charakteristika unterschiedlicher dokumentarischer und fiktionaler Fernsehsendungen zum Thema Medizingeschichte.

21 Zitiert in Rossmann, 2002 (Fn. 1), S. 9. 
chen Speicherung und Vermittlung von Erinnerungen an. Raumorientierte Speicherstrukturen gab es nicht nur im Motiv des „Erinnerungspalastes“ in der Kunstgeschichte, sondern auch in Form des Gedächtnistheaters, das historisches Wissen in Bildräumen ausdifferenziert. Im Gedächtnistheater des Italieners Camillo ist das Bild vor dem Text platziert. Es bestand aus einer Holzkonstruktion, die einem kleinen Rundtheater ähnelte. Der Innenraum konstituierte sich aus von zeitgenössischen Malern hergestellten Bildflächen, die in Geschosse und Segmente unterteilt waren. Hinter den Bildflächen fanden sich die nach Stichworten sortierten Texte mit den Wissensbeständen. Die Vermittlung sprachlicher Information war also der visuellen Vermittlung nachgeordnet. ${ }^{22}$

Im Prozess der Filmgeschichte hat sich ein komplexes Wechselverhältnis aus sprachlicher und visueller Information herausgebildet. Bedingt durch diese Komplexität weist der amerikanische Medienwissenschaftler Anton Kaes Spielfilmen eine besondere suggestive Bedeutung zu, die auch die Vermittlung historischer Inhalte beeinflusst:

„Spielfilme - anders als Reden oder wissenschaftliche Abhandlungen - sind auf-

grund ihrer mehrfach kodierten Materialität aus Bildern, Texten und Tönen kom-

plexe ästhetische Gebilde, die es erlauben, mit Ambivalenzen, Assoziationen und

Identifikationen zu spielen und dem Zuschauer mehrere Bedeutungen zugleich zu suggerieren. Damit gelingt es fiktionalen Filmen (öfter als dokumentarischen), im Zuschauer verborgene Wünsche und Ängste zu aktivieren, Phantasien freizusetzen, kollektive Stimmungslagen anzusprechen und Meinungen zu katalysieren. " 23 Das traditionelle Ordnungsprinzip der vom Bild ausgehenden, organisierten Erinnerung weicht aber auch in vielen Fernsehdokumentationen einer simultanen Vermittlung: Dabei vermittelt die Sprache die sachorientierte Information, während das Bild häufig auf die Darstellung des Handlungsortes oder die illustrierende Dekoration, etwa in Form der Abbildung alter Grafiken oder Schriftstücke, beschränkt bleibt. Feature und Dokudramen ${ }^{24}$ als Mischformen aus Fiktion und Dokumentation nutzen darüber hinaus den Erlebnischarakter etablierter filmischer Inszenierungsmuster des Historischen, z. B. in unterschiedlichen Formen szenischer Darstellung mit Schauspielern in historisch gestalteten Kulissen. Aus der sachorientierten Erinnerung wird hier die szenische Präsentation vergegenwärtigenden Miterlebens. Dabei stehen Personen als Akteure der historischen Ereignisse auch im Zentrum des Bildes. Sie bestimmen durch Dialog und Handlung die Bildbewegungen. Auf der visuellen Vermittlungsebene signalisieren im Vergleich zur heutigen Apparatemedizin altertümlich wirkende Requisiten, wie Laborausstattungen oder technische Instrumente, den Vergangenheitsbezug der Darstellung. Diese visuellen Darstellungsstereotypen sind den Zuschauern aus populären historischen Biopics von Ärzten wie „Das Leben des Dr. Sauerbruch“ (1954) vertraut.

\section{Medizingeschichte in unterschiedlichen Genres}

Im Verlauf der Fernsehgeschichte hat sich ein eigenes Ensemble aus Sendeformen der Geschichtsvermittlung herausgebildet, ${ }^{25}$ die auch die Vermittlung von Medizinge-

22 Vergleiche hierzu: Matussek, Peter (1999): Computer als Gedächtnistheater. In: Götz-Lothar Darsow (Hrsg.), Metamorphosen. Gedächtnismedien im Computerzeitalter, Stuttgart, S. 84f.

23 Kaes, Anton (1988): Geschichte im Film. Frankfurt am Main, S. 6.

24 Beide Formen wurden bereits im Hörfunk entwickelt.

25 Vergleiche hierzu: Bleicher, Joan Kristin (1993): Geschichte im Fernsehen. Zwischen Dokumentation und Identifikation. In: TheaterZeitSchrift, H. 33/34, S. $23-35$. 
schichte bestimmen. Das Fernsehen nutzt für die Vermittlung unterschiedlicher historischer Inhalte neben den Sendeformen des Informationsbereichs auch fiktionale Sendungsangebote: Dokumentation, Dokumentarspiel, Porträt, Spielfilm und Fernsehspiel. Viele dokumentarische Sendungen lehnen sich mit einer kleinteiligen Präsentation von Medizingeschichte an bestehende Aufmerksamkeitsstrategien an und nutzen Konventionen anderer Sendeformen, wie Magazin oder Nachrichten. Im Rahmen des dort praktizierten Kalenderjournalismus werden Themen aus dem Bereich Medizingeschichte etwa an Jubiläumsdaten platziert. Diese kleinteiligen Sendeformen finden sich beispielsweise in Kurzdokumentationen aus der Reihe „Rückblende“ oder einzelnen Beiträgen in Gesundheitsmagazinen wie „Visite“.

Die dem Zuschauer durch seine langjährige Seherfahrung vertrauten formalen und inhaltlichen Darstellungskonventionen dieser Sendeformen beeinflussen die Wirkung der vermittelten Inhalte. Diese Wirkung ist der Wirkung von Filmgenres vergleichbar. Die Definition von Genres als impliziten Vereinbarungen zwischen Produzenten und Zuschauern über die Art der Geschichte und ihren Informations- oder Erlebniswert verweist darauf, dass an der Bedeutungskonstruktion medialer Angebote unterschiedliche „Autoren“ beteiligt sind. Die Kombination genrespezifischer Darstellungselemente ist dabei ein entscheidender Faktor dieser Bedeutungskonstruktion. ${ }^{26}$

\subsection{Serielle Fenster in unterschiedliche Zeiträume der Medizingeschichte}

Erst in den parallelen Angeboten unterschiedlicher Sendeanstalten löst sich die klassische Linearität der Geschichtsschreibung als Konstruktionsprinzip des Fernsehens in ein Mosaik vielfältiger Zeitbezüge auf. Das Fernsehen als viel beschworenes Fenster in sonst unzugängliche Weltbereiche wird im Bereich der seriellen Präsentation von Medizin zum Fenster in unterschiedliche Zeiträume der Medizingeschichte.

Bei den Angeboten fiktionaler Serien sind beide Bereiche des Zeit-/Raumbegriffs bedeutsam, denn Produktionen aus unterschiedlichen Ländern sind nicht nur an unterschiedlichen Handlungsorten angesiedelt, in den Programmangeboten unterschiedlicher Sendeanstalten stehen aktuelle Produktionen der Arzt- und Krankenhausserien auch neben Produktionen aus unterschiedlichen Jahrzehnten der Programmentwicklung. Zur „Hoch-Zeit“ deutscher Arztserien in der zweiten Hälfte der neunziger Jahre, am 16. März 1998, erhielt der umschaltbereite Zuschauer Einblick in ganz unterschiedliche Zeiträume der Medizingeschichte: den Krankenhausbetrieb in der deutschen Provinz in den achtziger Jahren („Die Schwarzwaldklinik“, 1985), den amerikanischen Krankenhausbetrieb der neunziger Jahre („Chicago Hope“, 1996), in die deutsche Notaufnahme der neunziger Jahre („OP ruft Dr. Bruckner - Die besten Ärzte Deutschlands“, 1998), den Arbeitsalltag einer Ärztin in der amerikanischen Provinz des 18. Jahrhunderts („Dr. Quinn - Ärztin aus Leidenschaft“) und den Arbeitsalltag eines deutschen Arztes in der deutschen Provinz der fünfziger Jahre („Landarzt Dr. Brock“, 1967). Die Linearität der Geschichtskonstruktion des Fernsehens wird durch die gleichzeitige Platzierung verschiedener serieller Fernseherzählungen in ein Mosaik unterschiedlicher Zeit- und Raumbezüge aufgelöst.

Durch die gleich bleibenden Rollenmuster der Personalisierung (Arzt-Patient) und den stereotypen Erzählablauf von Krankheit und Heilung tritt die historische Zuord-

26 Vergleiche hierzu: Neal, Steve (1995): Questions of Genre. In: Barry Keith Grant (ed.), Film Genre Reader II, Austin, S. $159-183$. 
nung des Gezeigten hinter das gegenwärtige Miterleben zurück. So bleibt der Informationsgehalt der Thematisierung von Medizingeschichte in fiktionalen Sendeformen durch die besondere emotionale Wirkung begrenzt.

\subsection{Abhängigkeit der Genrewabl vom Zeitbezug der Inbalte}

Die Wahl des jeweiligen Genres ist auch abhängig von der Verfügbarkeit visuellen Quellenmaterials für ein historisches Thema. Vielfach werden vorfilmische Epochen, aus denen kein Quellenmaterial an bewegten Bildern zur Verfügung steht, in der szenischen Rekonstruktion des Spielfilms oder Fernsehfilms präsentiert. Dem Protagonisten/Antagonisten-Schema folgend erscheint etwa Paracelsus (1493-1541) in dem 1943 produzierten Film in der Regie von G. W. Pabst als gütiger Heiler, der für seine mittellosen Patienten die Normen seiner Zeit durchbricht; sein Antagonist ist als etablierter Arzt Teil der städtischen Hierarchie und am eigenen Machterhalt stärker interessiert als am Wohlergehen seiner Patienten. Die Verehrung der Patienten lässt den gütigen Arzt schnell als den göttlichen Helfer erscheinen. Damit erhält die historische Erzählung eine religiöse Bedeutungsdimension.

Auch die Form des Dokumentarspiels wird für Personen oder Ereignisse genutzt, für die keine entsprechenden Bildquellen zur Verfügung stehen. Im Dokumentarspiel finden sich Mischformen aus den dokumentarischen und fiktionalen Erzählweisen, die eine parallele Wirkung von Information und Erlebnisorientierung ermöglichen. War in den sechziger Jahren das Dokumentarspiel auf die aufwändig produzierte, detailgetreue szenische Rekonstruktion historischer Fakten bedacht, so hat sich mittlerweile auch eine weniger aufwändige Variante in der Darstellung von Medizingeschichte durchgesetzt. Ein Schauspieler stellt die Person des Arztes oder Forschers szenisch in Arbeitssituationen dar. Aus dem Off vermittelt eine Erzählerstimme Informationen über die Lebensgeschichte und verliest Zitate aus den Schriften des Porträtierten. Der Regisseur inszeniert historische Arbeit, indem er einen Schauspieler als Porträtierten während der Off-Erzählung am Schreibtisch sitzen lässt, wo er entweder in dicken Büchern blättert oder konzentriert in die Reagenzgläser eines Labors blickt.

Das etablierte Erzählprinzip historischer Dokumentationen mit ihrer linearen Chronologie der Ereignisse durchbricht ein Beitrag über die Berliner Charité an einigen Stellen zugunsten der simultanen Präsentation von Vergangenheit und Gegenwart im Splitscreen Verfahren. Hier sind im Kontrast die Veränderungen unmittelbar visuell anschaulich, die gleichzeitig auf der sprachlichen Vermittlungsebene abstrakt thematisiert werden.

Grundlegende Bildmotive der dokumentarischen Präsentation von Medizingeschichte finden sich auch in der Handlungsstruktur fiktionaler Sendeformen, die in der Gegenwart angesiedelt sind. Weiße Kittel, technische Geräte, OP-Säle oder Krankenhausflure signalisieren zeichenhaft Themenkomplex, Personal und Handlungsort des Geschehens. Allein visuelle Details entscheiden hier über die schnelle Unterscheidung des Historischen von der Gegenwart. Dabei ist das modische Aussehen der Kittel ein ebenso wichtiges Detail wie die Präsentation altertümlich anmutender medizinischer Gerätschaften und die Architektur der Krankenhäuser.

Je nach Sendungszusammenhang werden die historischen Bildmotive unterschiedlich verwendet. In fiktionalen Sendungen erhöhen die altertümlichen Gerätschaften als Requisiten den Grad der Bedrohung und damit den Erlebniswert der Handlung. Die von den Regisseuren intendierte Identifikation mit den Protagonisten und dem Handlungsverlauf fiktionaler Produktionen begünstigt das aktuelle Erleben, der Charakter infor- 
mativer Geschichtsvermittlung hingegen tritt dahinter zurück. Geschichten von Krankheiten und Heilen sollen ein Wechselbad der Gefühle wie Mitleid und Erleichterung auslösen. Das den Menschen gemeinsame Erleben von Krankheit soll die emotionale Betroffenheit der Zuschauer sichern.

\subsection{Historische Dokumentationen und Magazine}

Historische Dokumentationen folgen in ihrer grundlegend narrativen Vermittlungsstruktur den im Genre des Arztromans etablierten Erzählmustern der Geschichten vom Helfen und Heilen und kombinieren diese mit in der medialen Vermittlung etablierten Darstellungsmitteln der Inszenierung von Authentizität, etwa durch die Präsentation von Dokumenten oder die Einbindung des Erzählten in zeithistorische Bezüge. Auch im Bereich der Dokumentationen spielt die Stereotypenbildung auf der Handlungsebene eine zentrale Rolle: So bildet das Schicksal in Form der Krankheit den Ausgangspunkt vieler Erzählungen. Als Held fungiert der Arzt, der sich gegen das übermächtige Schicksal stellt und die Krankheit besiegt oder der in unermüdlicher Forschungsarbeit der Natur die Geheimnisse der Krankheit zu entreißen vermag. Der Wiederholungscharakter der seriellen Vermittlung unterstützt diese Konstruktion von Rollenmustern. Sendereihen wie „Das Jahrhundert der Chirurgen“ (1972) zeigen exemplarische Biographien von Forschern und Ärzten, die selbst in Monologen - etwa in Vorlesungen - als Vermittler ethischer Modelle auftreten. Mit der Personifizierung der Geschichte medizinischer Forschung kommt es zur Nutzung narrativer Darstellungsmuster für die Vermittlung faktischer Inhalte. Die Sendereihe „Das Jahrhundert der Chirurgen" zeigt aus programmgeschichtlicher Perspektive, dass bereits in den siebziger Jahren die Personalisierung der Informationsvermittlung die Grenzen zur fiktionalen Erzählung überschritt. „Diese Rückschau auf Höhepunkte der Medizingeschichte beschränkte sich nicht auf eine dokumentarische Rekonstruktion, sondern präsentierte die Pioniere in fiktionalisierter Form. "27 Ethische Intentionen als Handlungsmotivation des Forschers werden ergänzt durch menschliche Motivationen, wie Rettung und Heilung einzelner Menschen. Komplexe Forschungsentwicklungen werden auf das Handeln einzelner Personen reduziert und durch gleich bleibende Handlungsmuster von Helfen und Retten vereinfacht.

Es zeigt sich bei der Analyse unterschiedlicher Sendungsangebote, dass in Dokumentationen und Magazinen gleich bleibende Kerneinheiten - Krankheit als Problem, Forscher oder Heiler als personifizierte Instanzen der Lösung - je nach Themen- und Zeitbezug unterschiedlich zusammengefügt werden. Im Schatten der Halbgötter bleibt noch genug Licht für die Technik: Sie sonnt sich in den mythologischen Konstrukten des permanenten Fortschritts. Dokumentationen erzählen die Erfolgsgeschichten medizinischer Entwicklungen, die linear voranzuschreiten scheinen. Jeder Mediziner, so suggerieren es die Darstellungen, knüpft unmittelbar am Werk seines Vorgängers an und führt es durch eigene neue Erkenntnisse weiter. Irrwege, so scheint es in vielen Beiträgen, gab es in der medizinischen Forschung keine. Fehlerhafte Entwicklungen und die Opfer dieses Fortschritts sind lediglich in der gegenwartsbezogenen Berichterstattung zu sehen, so etwa jüngst bei dem Bayer-Skandal um die Nebenwirkungen eines Blutdruckpräparats.

27 Rosenstein, Doris (1998): Arzt- und Krankenhausserien. Profile eines Genres. In: Augenblick, H. 28: Die weiße Serie - Ärzte und Krankenhäuser im Fernsehen, Marburg, S. 16. 
In Beiträgen der politischen Magazine mit aktuellem Bezug jedoch entsteht ein Gegenbild mit neuen Rollenmustern. Medizingeschichte gerät hier - im Kontrast zu den Mythenbildungen der Spielfilme und Serien - häufig zu einem Antimythos der permanenten Fehlentwicklungen. Aus den Halbgöttern werden hier schnell skrupellose Verbrecher, die nicht das Motiv des Helfens, sondern das der Gewinnsucht verfolgen. Gesteigert wird die Wirkung der Darstellung durch eine Ansammlung von Opfern, der Patienten, die häufig in tränenreichen Selbstäußerungen ihr persönliches Schicksal als den Tätern hilflos ausgelieferte Menschen schildern.

Die dokumentarische Präsentation von Medizingeschichte im Fernsehen lehnt sich deutlich an Fortschrittsmodelle der Geschichtsschreibung an: Es scheint, als habe sich die Forschung linear und zielgerichtet fortbewegt, auch wenn immer nur Teilabschnitte thematisiert werden. Mit dieser Chronologie hält sich das Fernsehen die Berichterstattung für die Zukunft offen. Kontinuierlich lässt sich die Fortschrittsgeschichte der Medizin in den Sendungen weiter fortschreiben, jede neue Erzählung von Innovationen der Forschung und Entwicklung reiht sich ein in eine ungebrochene Kontinuität vergangener Entwicklungen, die das Medium fortlaufend konstruiert.

\section{Visuelle Darstellungsmittel der dokumentarischen Vermittlung}

Auf der visuellen Vermittlungsebene suggerieren unterschiedliche Darstellungsmotive den Charakter der Vermittlung von Geschichte. Je nach Quellenlage vorhandener Bildmaterialien verändert sich in den Sendungen der visuell sichtbare dokumentarische $\mathrm{Zu}$ griff auf den historischen Stoff. Dienen Schwarz-Weiß-Aufnahmen minderer Bildqualität als Suggestion authentischer Augenzeugenschaft, so verweisen Schriftquellen und Zeichnungen auf die faktische Übermittlung des Vergangenen. Doch der Einsatz dieser visuellen Quellen ist oft fragwürdig, wie es etwa ein Beitrag von Manfred Bauer über die Geschichte des Penicillins zeigt. Lässt man die ersten Minuten des Beitrags ohne Ton laufen, ist nicht erkennbar, welches Thema gerade behandelt wird. Die Bildebene zeigt unterschiedliche Formen medizinischer Behandlung, aber auch symbolisch erscheinende Figuren, die für den Zuschauer nicht entschlüsselt werden. Quellen werden so als Bildtapete für den sprachlichen Kommentar genutzt.

Andere Dokumentationen zeigen Schriftquellen, die in schneller Folge digital geblättert oder überblendet werden. Diese Form des Quelleneinsatzes zielt weniger auf konkrete Informationsvermittlung als auf einen bestimmten Effekt der Dynamisierung des Visuellen. Die Autorität des sichtbar gemachten historischen Quellenmaterials fungiert als vermeintlicher Authentizitätsbeweis für die vermittelten Inhalte. Der Historiker Siegfried Quandt bemerkt:

„Die historische Dokumentation lebt vom Original, und das im doppelten Sinn: erst das Dokument (sei es nun eine Filmaufnahme, ein Originalton oder eine Fotografie, ein Flugblatt, vielleicht auch nur eine Zeitung oder ein Brief) erleichtert dem Zuschauer mit seinem Erinnerungswert den Einstieg in ein Thema. Aktuelle Entwicklungen oder vergangene Ereignisse werden in ihrer Tragweite erst durch das historische Dokument deutlich und vermitteln durch ihre Authentizität das Gefühl des unmittelbaren Erlebnisses. “28

28 Quandt, Siegfried (1988): Einleitung. In: Guido Knopp/Siegfried Quandt (Hrsg.), Geschichte im Fernsehen. Ein Handbuch. Darmstadt, S. 12. 
Historische Grafiken, die Ärzte, Patienten, innere Organe und technisches Werkzeug präsentieren, finden sich in zahllosen medizinhistorischen Dokumentationen. Hier erlebt das Ordnungsprinzip des Gedächtnistheaters seine Renaissance. Hinter den Bildflächen ist das sprachlich fixierte Wissen verborgen. Allein die Off-Erklärungen suggerieren einen thematischen Zusammenhang der Aufnahmen, der durch ein digital simuliertes Umblättern oder durch die Überlagerung verschiedener Bildmotive noch verstärkt wird. Die Dokumentation zur Geschichte des Berliner Krankenhauses Charité (Regie: Rainer Meißle) nutzt in Ergänzung zur Off-Erklärung die direkte Erzählerinstanz eines Medizinprofessors (Prof. Dr. Gottfried Bogusch), der im Hörsaal einem doppelten Publikum, nämlich den Studenten und den Zuschauern vor den Bildschirmen, etwas zur frühen Geschichte der Pathologie erzählt. ${ }^{29}$ Die Vorlesung wird vom Regisseur als Erzählung in der Erzählung eingesetzt. Optisch wirksam werden die Objekte der Pathologie durch Charité-eigene historische Sammlungen deformierter Körper präsentiert. Krankheit gerät in dieser Darstellungsform zum konservierten Objekt der Schaulust. Die aus dem populären Film bekannten Deformationen werden hinter Glas für die Fernsehpräsentation reaktiviert und drohen durch ihre Eindrücklichkeit die Information der sprachlichen Erzählung über die Geschichte der Pathologie in den Hintergrund zu drängen.

Das Vermittlungsideal ist ein anderes: Der Reiz des Visuellen, so das Konzept der Regisseure, soll in die Reflexion des Dargestellten münden. Doch dieses Ideal wird von vielen historischen Sendungen nicht erreicht. Während in der Off-Moderation historische Fakten geschildert werden, zeigen die Bilder alte Kupferstiche oder Behandlungsinstrumente. Diese häufige Konkurrenz zwischen sprachlicher und visueller Vermittlung, die so genannte Text-/Bildschere ${ }^{30}$, verhindert nicht nur die gemeinsame Wirkung von sprachlicher und visueller Information, sie erschwert auch den Erinnerungswert des Präsentierten. Dieses Phänomen des Widerstreits verschiedener Informationsebenen findet sich in vielen Sendeformen des Fernsehens, die Geschichte präsentieren.

\section{Formen der Rezeptionssteuerung}

\subsection{Rezeptionssteuerung durch Sendeformen und Sendungstitel}

Mit der Ausstrahlung diverser fiktionaler und dokumentarischer Sendeformen zu unterschiedlichen Themen, Personen und Orten, nutzt das Medium Fernsehen den Themenkomplex Medizingeschichte für eine Bricolage historischer Versatzstücke mit unterschiedlichem Informations- und Erlebniswert. Der Prozess der Medizingeschichte wird in thematische Einzelelemente untergliedert, die sich in die allgemeine mediale Vermittlungsstruktur einfügen lassen. In dieser Bedeutungskonstruktion ist neben der Genrezuordnung auch die Titelgebung der Sendung ein zentraler Faktor der Steuerung

der Zuschauerwahrnehmung. In Titeln manifestieren sich die Funktions- und Erlebnisversprechen der jeweiligen Medienangebote. ${ }^{31}$ Sendereihen, die sich mit generellen his-

29 In der Dokumentation wechseln sich die Experten als Erzähler ab. Auch Vertreter anderer Disziplinen erzählen in Interviewform von der Geschichte ihres Faches an der Berliner Charité.

30 Wember, Bernward (1983): Wie informiert das Fernsehen? Ein Indizienbeweis. München, S. 47.

31 Vergleiche hierzu auch: Bleicher, Joan Kristin (2001): Nur ein toter Zuschauer ist ein guter Zuschauer. Programmverbindungen als Paratexte des Fernsehens. In: Navigationen, Jg. 1, Nr. 1, Siegen, S. $77-88$. 
torischen Phänomenen und Epochen befassen, verweisen zumeist bereits mit Titeln wie „Die Welt der 30er Jahre“ auf den Zeitraum, den sie behandeln, und vermitteln das Nutzungsversprechen Orientierung. Der im Titel signalisierte Anspruch, die „Welt“ zu präsentieren, schafft so den Eindruck von sinnhafter Übersichtlichkeit.

Andere Titel stellen durch die Angabe von Namen wie „Dr. Sauerbruch“ den Protagonisten ins Zentrum und verweisen so bereits in der Ankündigung auf die Personenbindung der Thematisierung von Medizingeschichte in dem jeweiligen Sendungsangebot. Auf diese Weise werden dem Zuschauer Identifikationsangebote signalisiert, die einen emotionalen Erlebniswert versprechen. So löst sich die Geschichtsdarstellung vom Schwerpunkt der Informationsvermittlung und nimmt einen eher unterhaltenden Charakter an.

\subsection{Rezeptionssteuerung durch Dramaturgie}

Der dramaturgische Einsatz unterschiedlicher Vermittlungsformen ist entscheidend für die Rezeption des präsentierten Themas aus dem Bereich Medizingeschichte. Dabei kommt insbesondere erzähltechnischen Verfahren der Personalisierung eine Schlüsselrolle zu, da sie über die Identifikation der Rezipienten die Akzeptanz bestimmter Inhalte zu erhöhen versuchen. Menschliche Identifikationsfiguren, sei es in der Rolle des Akteurs oder Opfers, sind ein wichtiger Faktor bei der Durchsetzung positiv besetzter Medizinbilder. Die kollektiv verbreitete Einschätzung, dass es die gesellschaftliche Funktion der Medizin sei, zur Verbesserung der gesundheitlichen Situation von Menschen beizutragen, ist eng an über längere Zeit hinweg etablierte, positiv bewertete ethische Konzepte geknüpft. Durch die Erinnerung an gleich bleibende Konzepte in unterschiedlichen Phasen der Geschichte wird die historische Entwicklung als Gültigkeitsgarant instrumentalisiert. Die Wirkung dieses Verfahrens wird durch die Personenbindung weiter gesteigert. Der Arzt als zentrale Instanz der Personifizierung positiver Rollenmuster bei der medialen Vermittlung von Medizingeschichte steht für die konservativen Wertemuster Güte, Aufopferungsbereitschaft und Selbstlosigkeit durch die für die narrative Heldenkonstruktion unersetzlichen Handlungselemente des Rettens und Heilens.

\section{Bindung zeitbezogener ethischer Konzepte an Rollenmuster}

Die Darstellung zeitabhängiger positiver und negativer Bewertung von Medizin ist an diese etablierten Rollenmuster der Thematisierung von Medizin geknüpft. Je nach Zeitbezug der Sendung lassen sich unterschiedliche ethische Konzepte der Rollenklischees unterscheiden. Negative ethische Konzepte manifestieren sich in der Präsentation von Rollenmustern skrupelloser Täter, aber auch der erkrankten Opfer. So finden sich Darstellungen imposanter Apparaturen mit einer Vielzahl von Schläuchen angeschlossen an Menschen, die ihrer menschlichen Selbstäußerungsmöglichkeiten beraubt nur noch als Versuchseinheiten fungieren. Ein anderes Stereotyp von Opferrolle schildert auf der Handlungsebene die Krankheit als durch ungesunden Lebenswandel selbst verschuldet. Dienen die Patienten in den medizinhistorischen Dokumentationen als bloße Körperapparate für die Forschung, so werden sie in fiktionalen Thematisierungen von Medizin häufig auf Schicksalsträger reduziert.

Diese in Themen und Rollengestaltung stereotype Präsentation von Medizingeschichte konstruiert einen medialen Wahrnehmungsrahmen, in den die aktuelle Berichterstattung eingeordnet wird. Die Vergangenheit ist Grundlage der Einordnung und Bewertung des Gegenwärtigen und der Fortschreibung des Künftigen. 


\section{Fazit}

Die im Beitrag gezeigte Thematisierung und Präsentation von Medizingeschichte im Fernsehen ist exemplarisch für die Darstellung von Geschichte im Fernsehen insgesamt. Das Fernsehen nutzt bestehende Gedächtnisräume von Literatur und Film als Inhalte. Historische Themen werden in unterschiedliche Sendeformen des faktenorientierten Erzählens überführt, deren Spektrum vom Fernsehfilm bis zur Magazinsendung reicht. Auch komplexe Zusammenhänge lassen sich durch die Bindung der Handlung an einzelne Personen und durch gleich bleibende Vermittlungsstrukturen vereinfacht und damit verständlicher darstellen.

Die Stereotypen der Vermittlung von Medizingeschichte beeinflussen auch die aktuellen Schemata der Thematisierung von Medizin. Krankheitsdiagnosen werden eng mit allgemeiner Lebensberatung, der Hilfe durch den Arzt, aber auch mit Konsumversprechen neuer medizinischer Forschungsentwicklungen, Produkte oder vorbeugender Gesundheitsartikel kombiniert. Unzählige Gesundheitsbeiträge in Magazinsendungen behandeln den kontinuierlichen Fortschritt medizinischer Entwicklungen und entlassen jede Woche aufs Neue ihre Zuschauer mit der stets gültigen Botschaft: „Du musst dein Leben ändern.“32 Das Medium weist im Appell das Individuum auf seine Lebenswelt zurück. Der Erfahrungsraum des Fernsehprogramms versucht, den Lebensraum der Zuschauer zu bestimmen. Medizinsendungen entsprechen in idealer Weise den Ratgeberfunktionen des Mediums.

32 Hier sind noch Spuren jener normativen Pädagogik vorhanden, die ansonsten in der Gesellschaft ausgestorben zu sein scheint. 